\title{
Atomic cluster collisions
}

\author{
Andrey V. Korol ${ }^{\mathrm{a}}$ and Andrey Solov'yov ${ }^{\mathrm{b}}$ \\ Frankfurt Institute for Advanced Studies, Goethe-Universität, Ruth-Moufang-Str. 1, 60438 Frankfurt am Main, Germany
}

Received 15 November 2012 / Accepted 27 November 2012

Published online 17 January 2013 - (C) EDP Sciences, Società Italiana di Fisica, Springer-Verlag 2013

\begin{abstract}
Atomic cluster collisions are a field of rapidly emerging research interest by both experimentalists and theorists. The international symposium on atomic cluster collisions (ISSAC) is the premier forum to present cutting-edge research in this field. It was established in 2003 and the most recent conference was held in Berlin, Germany in July of 2011. This Topical Issue presents original research results from some of the participants, who attended this conference. This issues specifically focuses on two research areas, namely Clusters and Fullerenes in External Fields and Nanoscale Insights in Radiation Biodamage.
\end{abstract}

\section{Introduction}

Recent years have witnessed the rapid and extensive development of a variety of experimental tools and of the corresponding theoretical models for the analysis of the structure, properties and dynamics of nuclear, atomic, molecular, biological and complex cluster systems. Frequently, these systems are studied by means of photonic, electronic, heavy particle and atomic collisions. More recent approaches include the employment of Free Electron Lasers to study atomic clusters. The attention has been devoted to dynamical phenomena, many-body effects taking place in clusters, nanostructures, molecular and biological systems, which include problems of fusion and fission, fragmentation, collective electron excitations, phase transitions and many others. Recently, the non-linear phenomena in coupling of electromagnetic radiation to atomic and molecular clusters were explored by new excitation possibilities in the VUV and X-ray regime for the first time. Both experimental and theoretical aspects of cluster physics uniquely placed between nuclear physics, on the one hand, and atomic, molecular and solid state physics on the other, were the subject of investigation.

The articles in this Topical Issue provide a snap shot of the current research activities in the field of structure formation and dynamics of nuclear, atomic, and molecular clusters, nanoobjects, ensembles of nanoparticles, nanostructures, bio-molecules, and bio-molecular systems. The contributions to this issue represent the studies both at the fundamental level of elementary mechanisms and at the more applied level which is necessary in numerous

\footnotetext{
${ }^{a}$ On leave from St. Petersburg State Marine Technical University, St. Petersburg, Russia.

b On leave from A.F. Ioffe Physical Technical Institute, St. Petersburg, Russia;

e-mail: solovyov@fias.uni-frankfurt.de
}

applications of nano- and bio- technology, material science and medicine.

The premier conference in this field, the international symposium on atomic cluster collisions (ISSAC) started in St. Petersburg, Russia in 2003 [1]. The second ISACC was held at the GSI, Darmstadt, Germany in 2007 [2]. Both first and second symposia were satellites of the international conferences on photonic electronic and atomic collisions (ICPEAC). The third ISACC returned to St. Petersburg, Russia in 2008. Then, in 2009 the symposium took place in Ann Arbor, again as a satellite meeting of the ICPEAC [3]. The sixth meeting will be held in China during the period July 19-22, $2013^{1}$. Initially the symposium was mainly focused on dynamics of atomic clusters, especially in atomic cluster collisions, but since then its scope has been widened significantly to include dynamics of nanosystems, bio-molecules, and macromolecules with the emphasis on the similarity of numerous essential clustering phenomena arising in different branches of physics, chemistry, and biology. The ISACC meetings gave rise to the series of another interdisciplinary conference, "Dynamics of Systems on the Nanoscale" (DySoN), which embraces a broad range of topics under a common theme. The first DySoN Conference took place in Rome, Italy in 2010 followed by DySoN 2012 held in St. Petersburg, Russia $^{2}$.

The ISACC 2011 brought together more than a hundred scientists in the field of atomic and molecular cluster physics from around the world. Below we briefly characterize the sessions which were held at the symposium.

The session "Structure and dynamics of clusters, nanoparticles" was devoted to recent advances in the understanding of structure and essential dynamic properties of selected cluster systems, nano-structures and confined systems. Both theoretical and experimental aspects were

\footnotetext{
1 http://fias.uni-frankfurt.de/isacc/overview/

2 http://fias.uni-frankfurt.de/dyson/home/
} 
discussed. The session "Electron and photon cluster collisions" was devoted to the problems of electron and photon collisions with various clusters and confined systems. Special attention in this session was given to the polarization and collective excitation effects. Both theoretical and experimental aspects of electron-cluster collisions were discussed.

The session "Ion-cluster collisions" was devoted to the physics of ionic collisions with fullerenes and metal clusters. It covered a broad spectrum of problems in this field from both experimental and theoretical points of view. The results of very recent measurements were reported.

The session "Clusters and bio-molecules in external fields: electric, magnetic, laser, etc." was focused on multiphoton processes occurring in clusters and complex molecules exposed to an intense XUV and VUV laser fields. Discussions on novel approaches in studying structural and dynamical phenomena in clusters, nanostructures, molecular and biological systems which become available with FELs operating in the wavelength regime from tens of nanometers down to even one Angström were carried out within the session "Cluster and bio-molecular research at FEL".

In the session "Cluster structure and dynamics on a surface" the problem of interaction of atomic clusters with a surface, of cluster deposition and formation at a surface as well as collision processes involving clusters deposited at a surface were discussed accompanied by a number of illustrative examples of both experimental and theoretical origin.

The session "Clustering in systems of various degrees of complexity" covered recent theoretical and experimental achievements in understanding fundamental problems of clustering, self-organization and structure-formation on the nanoscale in systems of various degree of complexity.

The session "Structure and dynamics of clusters and bio-molecules" included talks devoted to the methods of theoretical description of the structural properties of mixed metal clusters, of atomic, electronic and spin properties of free and supported clusters, as well as to the experimental techniques which allows one studying phase transition of self-assembled monolayers. Attention was paid to the fragmentation and protection of bio-molecules due to the effect of attaching water molecules. A talk was given on using intense femtosecond laser pulses as a tool for influencing and observing molecular dynamics.

\section{Atomic cluster science}

During last decades it was recognized, both experimentally and theoretically, that complex molecules and atomic clusters often possess unique properties, which make them a new object of physical research. The knowledge of the detailed electronic and ionic structure of single complex molecules and nanoclusters can be essential for various practical applications, such as the formation of new materials, nanostructures, in the design of drugs and biologically active species as well as for the understanding of fundamental issues, such as the functioning of quantum and thermodynamic laws in nanoscale systems or mechanisms for the formation of complex multi-atomic systems formation, self-assembly and functioning.

An atomic cluster can be defined as a group of atoms bound together by interatomic forces. There is no qualitative distinction between small clusters and molecules, except perhaps that the binding forces must be such as to permit the molecule to grow much larger by stacking more atoms or molecules of the same type if the system is to be called a cluster. As the number of atoms in the system increases, clusters acquire more and more specific properties, making them unique physical objects different from both single molecules and from the solid state.

There are many different types of atomic clusters: van der Waals clusters, metallic clusters, fullerenes, molecular, semiconductor, mixed clusters, and their shapes can depart considerably from the common spherical form: linear, spirals, fractal, etc. Usually, one can distinguish between different types of clusters by the nature of the forces between the atoms, or by the principles of spatial organization within the clusters. Clusters can exist in all forms of matter: solid state, liquid, gases and plasmas.

Clusters have been recognized as new physical objects with their own properties relatively recently as a result of several experimental successes. Discovery of the fullerenes [4], of the electronic shell structure in metal clusters $[5,6]$, the observation of plasmon resonances in metal clusters [7-9] and fullerenes [10,11], the formation of singly and doubly charged negative cluster ions [12] and many others.

Since the discovery of fullerenes by Kroto et al. [4], these compounds have been the objects of intensive experimental and theoretical investigations (see, e.g., [13]). The unique feature of a pristine fullerene $\mathrm{C}_{N}$ is that its carbon atoms form a stable cage with an empty interior. The mean radius of the void depends on the number $N$ of the atoms and varies from $3.5 \AA$ for $\mathrm{C}_{60}$ up to $\approx 31.5 \AA$ for a giant fullerene $\mathrm{C}_{4860}$ (see, e.g., [14]). Such a structure of fullerenes implies that they can enclose an atom, a group of atoms or even smaller fullerenes (so-called fullerene onions). The resulting endohedral complexes $\mathrm{A} @ \mathrm{C}_{N}$ are of current interest since their properties can be tuned by doping with atoms A (or molecules) of different types.

At present, the investigation of fullerenes and their derivatives, such as endohedral fullerenes, is a very active research field since they are proposed to be used in different fields of science and technology. One of the promising ideas is related to the possible application of fullerenes and their derivatives in medicine. They are potential carriers in mechanisms of drug and gene delivery $[15,16]$ and thought to have potential antiviral activity which might have strong implications on the treatment of HIV-infection [17]. Excitation of fullerenes placed in a biological medium by external radiation or incident heavy ions may lead to the generation of secondary electrons or reactive oxygen species which allows fullerenes to be potentially used as sensitizers in photodynamic therapy [18] and ion beam cancer therapy [19]. The possible medicinal applications of endohedral fullerenes include, for instance, 
their use in radio-immunotherapy for a stable encapsulation of radionuclides [20] and in radiology for enhancing imaging techniques $[21,22]$.

Due to the progress in laser technology [23] wellcontrolled short and intense laser pulses are available nowadays allowing one to explore light-induced dynamical phenomena far beyond the limits accessible with weak laser fields. Atomic and molecular nanometer-sized clusters in intense laser field are a nice playground to study the non-linear coupling of electromagnetic radiation to matter [24]. Whereas weak laser fields allow the analysis of photoionization, excited-state relaxation and structural modifications, the intense laser pulses lead to a variety of non-linear phenomena occurring in the systems. These include large-amplitude collective electron motion, above threshold ionization, production of highly charged atomic ions as well as of electrons with high kinetic energy, Coulomb explosion of the clusters, high harmonic generation and many others.

An even broader range of effects can be investigated for various cluster systems exposed to intense coherent light of a wavelength $\lambda$ ranging from tens of nanometers down to even one $\AA$. Intense and short laser pulses in the UV and X ray band can be delivered by Free Electron Lasers (FEL). The state-of-the-art FEL, recently commissioned at the SLAC National Accelerator, produces coherent soft and hard X-rays with peak brightness nearly ten orders of magnitude beyond conventional synchrotron sources and a range of pulse durations from 500 to less than ten femtoseconds [25]. Several other FEL X-ray facilities are either under construction or undergo advanced technical design work. With such characteristics this light source is capable of imaging the structure and dynamics of matter at atomic size and timescales [26]. Even more, it can be stated that "...FELs penetrate a terra incognita in light-matter interaction opening a new chapter in science throughout all disciplines, in physics, material science, chemistry or biology" [27].

The specific feature of atomic cluster physics is related to the fact that cluster properties provide a better understanding of the transition from the single atom or molecule to the solid state limit. Modern experimental techniques have made it possible to study this transition. By increasing the cluster size, one can observe the emergence of the physical features in the system, such as plasmon excitations, electron conduction band formation, superconductivity and superfluidity, phase transitions, fission and many more. Most of these many-body phenomena exist in solid state but are absent for single atoms.

\section{Clusters and fullerenes in external fields}

The properties of clusters and fullerenes can be studied by means of photon, electron and ion scattering which are the traditional tools for probing internal structure and dynamic properties various physical objects. During the last decade this toolkit has been also supplemented with the experimental studies of clusters interaction with intensive laser fields of a broad range frequencies.
A very important fundamental problem closely related to the mentioned applications is an adequate description of dynamic response of clusters and fullerenes to the action of external fields.

Contemporary software for the quantum-chemical calculations (e.g., Gaussian, GAMESS, NWChem, TURBOMOLE, etc.) provides an accurate quantitative description of the ground state of many-particle systems (clusters and fullerenes, in particular), and allows one to obtain information on geometrical and chemical properties of the system. Meanwhile, the description of dynamic properties, which play an important role in the processes of photoabsorption, elastic and inelastic scattering, electron attachment and photon emission, etc by means of such programs faces significant difficulties. Dynamic properties (for example, dynamic polarizability) are closely related to the response of a many-electron system to an external electromagnetic field. In many cases the properties are governed by a collective excitation of electrons and formation of the plasmon resonances in the excitation spectra [28]. In various systems, plasmon resonances lie either below the ionization threshold (in metal clusters) or above it (e.g., in fullerenes). To calculate the optical response of metal clusters can be performed with the help of quantum-chemical programs (see, e.g., [29,30]). However, it is a challenging task to apply these codes to calculate collective electron excitations in fullerenes, which lie in the continuous spectrum.

However, this can be achieved within simplified model approximations. The advantage of such approaches is that they allow one to overcome significant computational difficulties but at the same time take into account the essential features of the processes providing clear physical insight into the phenomena.

One of the well-known and widely used approaches is based on the jellium model [31]. The main feature of the model is that the detailed ionic structure of the system is smeared out and substituted by the uniform spherically symmetric distribution of the positive charge. Delocalized electrons of the system, moving in the field created by the uniform positive charge distribution, are considered within either the self-consistent Hartree-Fock (HF) or the local density (LDA) approximations. The jellium model was applied frequently to the description of ground state properties of metal clusters and fullerenes as well as to the investigation of photoexcitation and electron scattering processes in these systems (see, e.g., [32-39] and Ref. [28] for earlier references). Many-electron correlations can be taken into account, where it is necessary, using the perturbation theory, the random phase approximation with exchange (RPAE), or the time-dependent local density approximation (TDLDA). Model approaches provide quite accurate quantitative description of a variety of the collisional processes in which collective electron excitations (the plasmons) and/or polarization effects play a dominant role. From this viewpoint, the frameworks based on simplified but physically solid models have clear advantage in comparison with the standard quantumchemical methods. 
Delocalized electrons of atomic clusters may form two different types of collective excitations, namely the surface and volume plasmons [40-42]. The dipole surface plasmon is responsible for the formation of the giant resonance in photoabsorption spectra of metal clusters [43,44] and fullerenes [11], and plays also an important role in the process of inelastic scattering of electrons [28]. The electron impact ionization of metal clusters revealed the existence of the volume plasmon which has a higher resonance frequency and is essential for the formation of the ionization cross section [42].

While the photoionization experiments allow one to investigate only optically allowed dipole excitations, the electron energy loss (EEL) experiments, where an electron scattered at a certain angle is detected, probe plasmon excitations of higher angular momenta. The first experimental observation of plasmon excitations in the EEL spectra of gaseous $\mathrm{C}_{60}$ [45] revealed a maximum at about $22 \mathrm{eV}$, which was consistent with previous theoretical predictions [10] and with the later experimental observations $[11,46]$ of the surface plasmon in the photoionization spectra.

Theoretical investigations of fast electrons scattering on metal clusters and fullerenes, carried out the RPAE as well as in the plasmon resonance approximation, revealed the existence of the diffraction phenomena (see Ref. [28]). The first experimental observation of the electron diffraction on $\mathrm{C}_{60}$ was reported in reference [47]. It was shown that plasmon modes of different angular momenta provide dominating contributions to the differential cross section at different electron scattering angles. In reference [48], the plasmon resonance approximation was applied to describe the dynamic response of atomic clusters in the processes of radiative electron capture, polarizational bremsstrahlung and multiphoton excitation.

Recent experiments on photoionization of neutral [49] and charged [46] $\mathrm{C}_{60}$ molecules revealed the presence of the second collective resonance at about $40 \mathrm{eV}$ which initially was interpreted as a volume plasmon [46]. Later on it was argued that the second resonance was associated not with the volume plasmon but with the antisymmetric mode of the surface plasmon [50] (see also [51]). While only two surface plasmons can be excited by the uniform field of an electromagnetic wave in the process of photoionization, the volume plasmon may be formed in the system under the exposure to a non-uniform field of the charged projectile.

Another type of atomic clusters, endohedral fullerenes, have been investigated experimentally and theoretically during the last decade. These complexes have an advantage over the pristine fullerenes since their properties can be tuned by doping with atoms or molecules of different types. As the ability to insert atoms or molecules into carbon cages increased, so too has the interest in these endohedral systems grown (see, for example, the reviews $[22,52])$. One of the ways to explore the properties of endohedral systems is to use spectroscopic means. If the confining effect of the fullerene cage on the optical properties of the endohedral species can be understood, then this may provide means of predicting the properties of the whole system.

The presence of a relatively compact object inside the fullerene cage give rise to the effects which do not exist either in pristine fullerenes or isolated atoms and molecules. For example, the photoionization cross section of the encaged atom acquires specific resonance-like behaviour. These so-called confinement resonances appear as a result of interference between a direct wave of the photoelectron escaping the atom and the waves due to scattering from the atoms of the cage [53]. Depending on the photoelectron momentum the interference can be constructive or destructive. Thus, the spectrum of the encaged atom acquires additional oscillations as compared to the free atom. The cage-induced oscillations have the same nature as the extended X-ray absorption fine-structure (EXAFS) for solid-state systems. To date, a number of theoretical investigations have been carried out studying confinement resonances in various processes, including photoionization and electron scattering. These and other related topics have been recently reviewed in reference [54]. Recently, the existence of the confinement resonances in the photoionization spectrum has been confirmed experimentally [55]. It was done for $\mathrm{Xe} @ \mathrm{C}_{60}$. Compared to other noble-gas endohedral complexes this system is mostly stable to the thermal vibrations of the atom which may completely destroy the resonance picture [56].

The fullerene electron shell can noticeably affect the decay of a vacancy in the encaged atom $[57,58]$. Dramatic broadening of the atomic Auger decay peaks due to the presence of the fullerene cage was experimentally measured for $\mathrm{Sc}_{3} \mathrm{~N} @ \mathrm{C}_{80}^{+}$in the photon energy range $30 \ldots 45 \mathrm{eV}$ [59]. Later, theoretical explanation of this effect was given [60]. It was shown that the widths of the Auger transitions in $\mathrm{Sc}^{2+}$ become strongly enhanced (by the factor of up to $10^{3}$ ) due to (a) non-central position of the ion in the cage, (b) strong multipole polarizability of the fullerene in vicinity of the second surface plasmon [50].

When an endohedral system is exposed to an external electromagnetic field, the fullerene, being dynamically polarized, screens the confined atom. The field at the atom may be stronger or weaker than the external field depending on its frequency $\omega$. Therefore, the photoionization cross section, $\sigma_{\mathrm{A} @ \mathrm{C}_{60}}(\omega)$, of the confined atom can differ from that $\sigma_{\mathrm{A}}(\omega)$ of the free atom in the same external field. This phenomenon was considered for the first time in [61] and was called a "dynamical screening". The cited paper has stimulated theoretical activity in the field. The dynamical screening was considered by using quantum mechanical approach $[62,63]$, as well as by applying the model semi-classical approach $[64,65]$ which turned out to be as accurate as much more sophisticated TDLDA-based calculations $[66,67]$.

\section{Nanoscale insights in radiation biodamage}

Besides significant fundamental interest, both experimental and theoretical, to the collisional processes involving metal clusters, nanoparticles and fullerenes, there 
is a number of potential applications based on these phenomena.

One of the important medical problems, which is still to be solved, is the cancer treatment. Modern methods of treatment include photodynamic therapy and ion beam cancer therapy (IBCT). These techniques are based on the fact that high-energy radiation, interacting with living tissue, produces structural and chemical modifications which affect biological functions and cause death of tumor cells. The modifications occur via the production of various intermediate species, in particular, secondary electrons.

It is widely accepted that the major part of damage done by external radiation or incident heavy ions is related, directly or indirectly, to the secondary electrons produced by ionization of the medium [68]. These electrons may interact with parts of DNA molecules in the cell nuclei, generate other secondaries, such as other electrons and radicals, which can interact with DNA. Increase of a number of DNA double strand breaks and, correspondingly, the probability of the tumor cell death can be achieved by injecting many-electron nanoparticles which play a role of sensitizers.

At present, considerable interest is in using metallic nanoparticles as photosensitizers in the cancer treatment with ionizing radiation [69-71]. Since the first experimental evidence of gold nanoparticles radiosensitization [72], this activity has been expanding and a number of new experiments on the sensitization with various metallic nanoparticles have been performed recently $[73,74]$.

To describe properly the influence of sensitization on the mechanism of DNA double strand breaks, it is necessary to know the electronic spectrum of secondary electrons propagating through the medium. In reference [75] spectra of secondary electrons generated in water by energetic ions have been obtained. For the medium with injected sensitizers (fullerenes, in particular) it is essential to carry out calculations of the secondary electron spectra.

The scientific interest in obtaining a deeper understanding of radiation damage is motivated by the development of ion-beam cancer therapy and other applications of ions interacting with biological targets [76-78]. A number of important scientific questions, especially related to DNA damage assessment on the molecular level, have not yet been resolved. Therefore, recently this field has attracted much attention from the scientific community. There are series of conferences devoted to these subjects $[19,77]$.

Among these studies is the multiscale approach to the assessment of radiation damage induced by irradiation with ions $[68,79]$. This method combines effects happening on a number of spatial, temporal, and energy scales. The goal of the multiscale approach is to quantitatively relate these effects to the cell damage and calculate the relative biological effectiveness and other macroscopic quantities. The main issues addressed by the multiscale approach are ion stopping in the medium, the production and transport of secondary electrons produced as a result of ionization and excitation of the medium, the interaction of secondary particles with biological molecules, the most important being DNA, the analysis of induced damage, and the evaluation of the probabilities of subsequent cell survival or death.

The milestones in the development of the multiscale approach were the calculations of the Bragg peak, the estimation of DNA strand breaks yields by secondary electrons, calculation of the radial dose, and assessment of the complex DNA damage [68]. A special investigation is devoted to DNA damage as a result of thermomechanical effects caused by ions [68,80-82]. These effects follow a dynamical change in temperature and pressure within a small cylinder surrounding the ions path leading to a cylindrical shock wave, which may rupture bonds in DNA molecules and also provides an effective mechanism of transport of reacting species, which may damage a DNA molecule several decades of nm away from the path. The understanding of such a possibility evolved from the estimates of the temperature increase in the medium as a result of ion propagation [80] to the analysis of thermal and pressure spikes in liquid water and further to the analysis of the shock wave in the medium [81] and modeling of its action on biological molecules [82].

\section{References}

1. Latest Advances in Atomic Clusters Collision: Fission, Fusion, Electron, Ion and Photon Impact, edited by J.-P. Connerade, A.V. Solov'yov (Imperial College Press, London, 2004), pp. 1-383

2. Latest Advances in Atomic Clusters Collision: Structure and Dynamics from the Nuclear to the Biological Scale, edited by J.-P. Connerade, A.V. Solov'yov (Imperial College Press, London, 2008), pp. 1-442

3. Proceedings of ISACC 2009, in AIP Conf. Proceedings, edited by A.V. Solov'yov, E. Surdutovich (2009), Vol. 1197

4. H.W. Kroto et al., Nature 318, 162 (1985)

5. W.D. Knight, K. Clemenger, W.A. de Heer, W.A. Saunders, M.Y. Chou, M.L. Cohen, Phys. Rev. Lett. 52, 2141 (1984)

6. W. Ekardt, Phys. Rev. B 29, 1558 (1984)

7. C. Bréchignac, Ph. Cahuzac, F. Carlier, J. Leygnier, Chem. Phys. Lett. 164, 433 (1989)

8. K. Selby, M. Vollmer, J. Masui, V. Kresin, W.A. de Heer, W.D. Knight, Phys. Rev. B 40, 5417 (1989)

9. K. Selby, V. Kresin, J. Masui, M. Vollmer, W.A. de Heer, A. Scheidemann, W.D. Knight, Phys. Rev. B 43, 4565 (1991)

10. G.F. Bertsch, A. Bulgac, D. Tomanek, Y. Wang, Phys. Rev. Lett. 67, 2690 (1992)

11. I.V. Hertel, H. Steger, J. de Vries, B. Weisser, C. Menzel, B. Kamke, W. Kamke, Phys. Rev. Lett. 68, 784 (1992)

12. A. Herlert, S. Krückeberg, L. Schweikhard, M. Vogel, C. Walther, Phys. Scr. T80, 200 (1999)

13. Handbook of Nanophysics: Clusters and Fullerenes, edited by K.D. Sattler (CRC Press, 2010)

14. B.-C. Wang, H.-W. Wang, J.-C. Chang, H.-C. Tso, Y.-M. Chou, J. Mol. Struct. (Theochem) 540, 171 (2001)

15. P. Anilkumar et al., Curr. Med. Chem. 18, 2045 (2011)

16. J.B. Melanko, M.E. Pearce, A.K. Salem, in Nanotechnology in Drug Delivery, edited by M.M. de Villiers, P. Aramwit, G.S. Kwon (Springer, New York, 2009), p. 105

17. P. Bakry et al., Int. J. Nanomed. 2, 639 (2007) 
18. P. Mroz et al., in Medicinal Chemistry and Pharmacological Potential of Fullerenes and Carbon Nanotubes, edited by F. Cataldo, T. Da Ros (Springer Science + Business Media B.V., New York, 2008), p. 79

19. 1st Nano-IBCT Conference 2011 Radiation Damage of Biomolecular Systems: Nanoscale Insights into Ion Beam Cancer Therapy, edited by B.A. Huber, C. Malot, A. Domaracka, A.V. Solov'yov, J. Phys.: Conf. Ser. 373 (2012)

20. M.D. Diener, J.M. Alford, S.J. Kennel, S. Mirzadeh, J. Am. Chem. Soc. 129, 5131 (2007)

21. P.P. Fatouros et al., Radiology 240, 756 (2006)

22. L. Dunsch, S. Yang, Small 3, 1298 (2007)

23. Femtosecond Laser Pulses: Principles and Experiments, in Advanced Texts in Physics, edited by C. Rullière, 2nd edn. (Springer, New York, 2005)

24. Th. Fennel, K.-H. Meiwes-Broer, J. Tiggesbäumker, P.-G. Reinhard, P.M. Dinh, E. Suraud, Rev. Mod. Phys. 82, 1793 (2010)

25. P. Emma et al., Nat. Photon. 4, 641 (2010)

26. A. Barty et al., Nat. Photon. 6, 35 (2012)

27. J. Ullrich, ISACC 2011, Book of Abstracts (2011), p. 46

28. A.V. Solov'yov, Int. J. Mod. Phys. 19, 4143 (2005)

29. A. Rubio, J. A. Alonso, X. Blase, L.C. Balbas, S.G. Louie, Phys. Rev. Lett. 77, 247 (1996)

30. I.A. Solov'yov, A.V. Solov'yov, W. Greiner, J. Phys. B 37, L137 (2004)

31. W. Ekardt, Phys. Rev. B 29, 1558 (1984)

32. K. Jänkälä, M.-H. Mikkelä, M. Huttula, J. Phys. B 44, 105101 (2011)

33. K. Jänkälä et al., Phys. Rev. Lett. 107, 183401 (2011)

34. O. Kidun, N. Fominykh, J. Berakdar, Comp. Mater. Sci. 35, 354 (2006)

35. A.K. Belyaev et al., Int. J. Quant. Chem. 107, 2781 (2007)

36. M.E. Madjet, H.S. Chakraborty, J.M. Rost, S.T. Manson, J. Phys. B 41, 105101 (2008)

37. A.K. Belyaev et al., Phys. Scr. 88, 048121 (2009)

38. A.V. Verkhovtsev et al., J. Phys. B 45, 215101 (2012)

39. A.V. Verkhovtsev et al., J. Phys. B 45, 141002 (2012)

40. W.A. de Heer, Rev. Mod. Phys. 65, 611 (1993)

41. U. Kreibig, M. Vollmer, Optical Properties of Metal Clusters (Springer-Verlag, 1995)

42. L.G. Gerchikov, A.N. Ipatov, R.G. Polozkov, A.V. Solov'yov, Phys. Rev. A 62, 043201 (2000)

43. H. Haberland, Clusters of Atoms and Molecules: Theory, Experiment, and Clusters of Atoms, Springer Series in Chemical Physics (Springer-Verlag, 1994)

44. W. Ekardt, Metal Clusters, Wiley Series in Theoretical Chemistry (Wiley, 1999)

45. J.M. Keller, M.A. Coplan, Chem. Phys. Lett. 193, 89 (1992)

46. S.W.J. Scully et al., Phys. Rev. Lett. 94, 065503 (2005)

47. L.G. Gerchikov, P.V. Efimov, V.M. Mikoushkin, A.V. Solov'yov, Phys. Rev. Lett. 81, 2707 (1998)

48. J.-P. Connerade, A.V. Solov'yov, Phys. Rev. A 66, 013207 (2002)

49. A. Reinköster et al., J. Phys. B 37, 2135 (2004)

50. A.V. Korol, A.V. Solov'yov, Phys. Rev. Lett. 98, 179601 (2007)
51. S.W.J. Scully et al., Phys. Rev. Lett. 98, 179602 (2007)

52. A. Rodríguez-Fortea, N. Alegret, A.L. Balch, J.M. Poblet, Nat. Chem. 2, 955 (2010)

53. J.-P. Connerade, V.K. Dolmatov, S.T. Manson, J. Phys. B 33, 2279 (2000)

54. V.K. Dolmatov, in Theory of Confined Quantum Sytems: Part 2, edited by J.R. Sabin, E. Brändas, Adv. Quant. Chem. (Academic Press, New York, 2009), Vol. 58, p. 13

55. A.L.D. Kilcoyne et al., Phys. Rev. Lett. 105, 213001 (2010)

56. A.V. Korol, A.V. Solov'yov, J. Phys. B 43, 201004 (2010)

57. V. Averbukh, L.S. Cederbaum, Phys. Rev. Lett. 96, $053401(2006)$

58. M.Ya. Amusia, A.S. Baltenkov, Phys. Rev. A 73, 063206 (2006)

59. A. Müller et al., J. Phys.: Conf. Ser. 88, 012038 (2007)

60. A.V. Korol, A.V. Solov'yov, J. Phys. B 44, 085001 (2010)

61. J.-P. Connerade, A.V. Solov'yov, J. Phys. B 38, 807 (2005)

62. M.Ya. Amusia, A.S. Baltenkov, Phys. Rev. A 73, 062723 (2006)

63. M.Ya. Amusia, A.S. Baltenkov, Phys. Lett. A 360, 294 (2006)

64. S. Lo, A.V. Korol, A.V. Solov'yov, J. Phys. B 40, 3973 (2007)

65. S. Lo, A.V. Korol, A.V. Solov'yov, Phys. Rev. A 79, 063201 (2009)

66. M.E. Madjet, H.S. Chakraborty, S.T. Manson, Phys. Rev. Lett. 99, 243003 (2007)

67. H.S. Chakraborty, M.E. Madjet, J.-M. Rost, S.T. Manson, Phys. Rev. A 78, 013201 (2008)

68. E. Surdutovich, A.V. Solov'yov, J. Phys.: Conf. Ser. 373, 012001 (2012)

69. S. Bosi, T. Da Ros, G. Spalluto, M. Prato, Eur. J. Med. Chem. 38, 913 (2003)

70. W. Chen, J. Zhang, J. Nanosci. Nanotechnol. 6, 1159 (2006)

71. K. Kobayashi, N. Usami, E. Porcel, S. Lacombe, C. Le Sech, Mutation Res. 704, 123 (2010)

72. J.J. Hainfeld, D.N. Slatkin, H.M. Smilowitz, Phys. Med. Biol. 49, N309 (2004)

73. E. Porcel et al., Nanotechnology 21, 085103 (2010)

74. S.J. McMahon et al., Sci. Rep. 1, 18 (2011)

75. E. Scifoni, E. Surdutovich, A.V. Solov'yov, Phys. Rev. E 81, 021903 (2010)

76. D. Schardt, T. Elsässer, D. Schulz-Ertner, Rev. Mod. Phys. 82, 383 (2010)

77. I. Baccarelli, F.A. Gianturco, E. Scifoni, A.V. Solov'yov, E. Surdutovich (eds.), Topical issue on Molecular level assessments of radiation biodamage, Eur. Phys. J. D 60, 1 (2010)

78. A. Kumar, M. Sevill, Chem. Phys. 110, 7002 (2010)

79. A.V. Solov'yov, E. Surdutovich, E. Scifoni, I. Mishustin, W. Grainer, Phys. Rev. E 79, 011909 (2009)

80. M. Toulemonde, E. Surdutovich, A.V. Solov'yov, Phys. Rev. E 80, 031913 (2009)

81. E. Surdutovich, A.V. Solov'yov, Phys. Rev. E 82, 051915 (2010)

82. A.V. Yakubovich, E. Surdutovich, A.V. Solov'yov, Nucl. Instrum. Methods Phys. Res. B 279, 135 (2012) 